\title{
Valoración del alumnado sobre distintos formatos de vídeos utilizados en docencia inversa en prácticas de laboratorio.
}

J.A. Gómez-Tejedor ${ }^{a}$, R. Martínez Salaa , J.M. Meseguer-Dueñas ${ }^{a}$, J. Molina-Mateo ${ }^{a}$, S. Quiles Casado $^{a}$, J. Riera ${ }^{a}$, M.A. Serrano-Jareño ${ }^{a}$, I. Tort-Ausina ${ }^{a}$ y A. Vidaurre ${ }^{a}$

${ }^{a}$ ETS de Ingeniería del Diseño, Universitat Politècnica de València (SPAIN)

\begin{abstract}
The students'opinion about the videos used in the flipped teaching in laboratory sessions is analized in this work. A survey with 7 multiple choice questions and one open question has been performed, obtanining 54 replies, from a total number of 155 students whi are attending the laboratory sessions. Students were asked about their preference between videos and written material (with some images), and about their preference between the three types of videos pffered: screencast (one of them including the image of the teacher and the other one with his/her voice only), and "Polimedia". Results have shown that students prefer video files instead of written docuements when they have to prepare their laboratory sessions. Moreover, there is a statistically significant preference for the videos where the image of the professor is shown. On the other hand, despite the format of video "Poliformat" has been the best valuated, there are certain aspects to be added, as the valuation thay made of the other types of videos (which is also good), or the critrica comments on this format shown in the open question of the survey.
\end{abstract}

Keywords: videos, Polimedia, screencast, laboratory, flipped teaching

\section{Resumen}

En este trabajo se analiza la opinión de los estudiantes sobre los vídeos utilizados en la docencia inversa de prácticas de laboratorio. Se ha realizado una encuesta con 7 preguntas de respuesta múltiple y una de respuesta abierta, contestada por 54 alumnos de los 155 que asisten al laboratorio. En ella se pregunta sobre la valoración del video frente documentos de texto e imagen, y sobre tres modelos de vídeo: dos de ellos responden al modelo de "screencast" (en uno de ellos está presente la imagen del profesor y en otro no) y el tercero es un "Polimedia". Los resultados indican que los estudiantes, en general, prefieren archivos de video a documentos de texto para la preparación de las prácticas de laboratorio. Además, se observa una preferencia estadísticamente significativa a favor de los videos en los que se muestra la imagen del profesor. Por otra parte, aunque el formato de video "Polimedia" ha sido el mejor valorado, hay ciertos matices a destacar, como es la valoración que hacen de los otros dos tipos de vídeos, que también es buena, o los comentarios críticos con este formato mostrados en la pregunta de respuesta abierta.

Palabras clave: videos, Polimedia, screencast, laboratorio, docencia inversa 


\section{Introducción y objetivos}

Un screencast es un video tutorial que combina información visual (capturada directamente del monitor del ordenador) y de audio y es accesible a través de internet (Peterson, 2007). Es una herramienta muy versátil para complementar la enseñanza de materias basadas en habilidades, como la física o las matemáticas (Ahmad \& Doheny, 2014), (Jordan, Loch, Lowe, Mestel, \& Wilkins, 2012). Puede usarse como complemento de la enseñanza presencial y son especialmente útiles en la aplicación de la metodología de clase inversa. En la metodología de clase inversa las sesiones tradionales de clases y trabajo en casa están invertidas (Zaphiris \& Ioannou, 2015). El tiempo de clase se optimiza porque el profesorado se dedica a resolver dudas en lugar de presentar toda la información (Lasry, Dugdale, \& Charles, 2014), (Blasco, Lorenzo, Sarsa, \& Sarsa, 2016). En el caso del laboratorio se optimiza el tiempo porque se empieza a trabajar desde el momento en que se entra en el laboratorio, sobre algo que ya se ha asimilado (al menos parcialmente).

Screencast ofrece múltiples beneficios para preparar el material de estudio. Tanto en el caso de que se use como complemento de las clases presenciales como de material de preparación de las sesiones de clase inversa, los estudiantes deben disponer, con antelación a la clase del material adecuado. Screencast ofrece la ventaja de adpatarse a cada estudiante permitiendo seleccionar la cantidad de información que quieren ver y con qué ritmo; ellos deciden si van hacia adelante, pausan o repiten un fragmento de screencast.

Para conseguir screencast efectivos es preciso prestar atención a su diseño, tanto formal como de contenidos (Sugar, Brown, \& Luterbach, 2010), (Oud, 2009). Se ha demostrado que los screencast cortos con un objetivo concreto producen mejores resultados de aprendizaje (Jordan et al., 2012), (Sutton-Brady, Scott, Taylor, Carabetta, \& Clark, 2011). En general, la combinación de gráficos, vídeos grabados en el laboratorio, con la narración del profesor, produce una mejora en proceso de aprendizaje cuando es comparado con el texto en formato tradicional (Mayer, 2014). Dunn et al. (Dunn, McDonald, \& Loch, 2015) han comprobado que los estudiantes utilizan activamente los screencast y lo consideran un importante material que les ayuda a estudiar.

En este trabajo se analiza la opinión de los estudiantes sobre una serie de vídeos desarrollados por los profesores de las asignatura de física y electricidad de primer curso de la Universitat Politècnica de València (UPV, http://www.upv.es) del Grado en Ingeniería Electrónica y Automática Industrial en la ETS de Ingeniería del Diseño (ETSID). Los vídeos, junto con textos en formato tradicional, sirven como material de apoyo para preparar las sesiones de laboratorio, impartidas en la modalidad de docencia inversa. Se han elaborado tres modelos de vídeo: dos de ellos responden al modelo habitual de screencast (en uno de ellos está presente la imagen del profesor y en otro no) y el tercero es un Polimedia (sistema diseñado en la UPV para la creación de contenidos multimedia como apoyo a la docencia presencial, que abarca desde la preparación del material docente hasta la distribución a través de distintos medios a los destinatarios). Todos tienen una duración similar, entre 10 y 15 minutos, y abordan contenidos de similar dificultad.

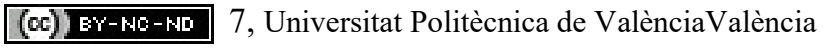


El objetivo de este trabajo es comprobar si son ciertas las siguientes hipótesis nulas:

H1. Para preparar los contenidos de las sesiones de laboratorio los estudiantes prefieren el material en vídeo al formato tradicional.

H2. Los estudiantes consideran que la presencia de la imagen del profesor en la pantalla ayuda a fijar las ideas fundamentales de la práctica.

H3. Los estudiante prefieren los vídeos en formato Polimedia a los screencast.

\section{Desarrollo de la innovación}

La metodología de docencia inversa se ha aplicado en las prácticas de laboratorio de la materia Física (asignaturas de Física y Electricidad), en los tres grupos de primer curso del Grado en Ingeniería Electrónica y Automática Industrial, en la ETS de Ingeniería del Diseño (ETSID) de la UPV. Se han realizado un total de 14 prácticas a lo largo del curso académico.

El material de que disponen los alumnos para preparar y realizar las prácticas se ha organizado utilizando la herramienta Lessons de Poliformat. Un valor añadido a este material es el de que sus contenidos son multilingües, utilizando las dos lenguas oficiales, valenciano y castellano, y el inglés.

La organización, en páginas, es la siguiente:

- Calendario + Instrucciones, para antes de iniciar las sesiones de prácticas, con la información general sobre las prácticas, calendario, método de evaluación...

- Para cada práctica de laboratorio:

- Antes de la sesión de la práctica, disponen de toda la información necesaria para iniciar la práctica de forma autónoma desde el momento en el que acceden al laboratorio: material escrito y vídeos que hemos elaborado con una explicación detallada de los objetivos y metodología a seguir durante la sesión. Estos vídeos y su diferente tipología son el objeto de análisis en el presente trabajo.

- Durante la sesión de la práctica, disponen de documentos de ayuda que pueden necesitar mientras están realizando la práctica, y el enlace a una actividad donde deberán subir la hoja de cálculo que han utilizado para recoger los datos experimentales durante la práctica.

- Después de la sesión de la práctica, disponen del enlace a la actividad donde deben subir la memoria de la práctica realizada para su evaluación.

En las sesiones de laboratorio, de dos horas de duración, los estudiantes comienzan su trabajo desde que acceden al laboratorio y el profesor se dedica a tutorizar y complementar su trabajo, y a discutir sus resultados. Esto supone una diferencia de unos 30 minutos más de tiempo efectivo de trabajo de los estudiantes, si lo comparamos con el aprovechamiento que se hacía con la metodología tradicional, en cursos anteriores.

Para introducir el trabajo en el laboratorio hemos realizado un conjunto de vídeos donde se presenta cada una de las prácticas a realizar, se plantean las hipótesis a comprobar, los fundamentos teóricos, los montajes experimentales con detalle (en algunos casos con fotografías y en otros grabados sobre el material que se van a encontrar los alumnos al realizar la práctica), y el tratamiento posterior de los datos experimentales. Los vídeos tienen tres formatos diferentes: 
- Screencast en el que la presentación va acompañada únicamente por la voz del profesor (figura 1).

- Screencast en el que se incluye la imagen del profesor en un marco incrustado en la imagen de una presentación (figura 2).

- Formato Polimedia: se trata de un sistema diseñado en la UPV para la creación de vídeos multimedia de contenido educativo de alta resolución. Consisten en una grabación en estudio, tras la cual se realiza una edición por parte del personal técnico, dando lugar a un archivo en formato MP4, disponible online, con la presentación combinada con la imagen del profesor (figura 3).

En este trabajo evaluamos la opinión de los alumnos sobre la utilización de estos tres tipos de vídeos, en el contexto de ser material de presentación de prácticas de laboratorio.

En los vídeos se han utilizado presentaciones digitales, hojas de cálculo, imágenes fijas y vídeos capturados mediante smartphone de partes de la explicación que lo necesitaban. Los screencast se han grabado y editado por los propios profesores mediante programas de software libre, y los Polimedia mediante los medios y técnicos de la UPV. Todos los vídeos están disponibles en el repositorio de la UPV (media.upv.es).

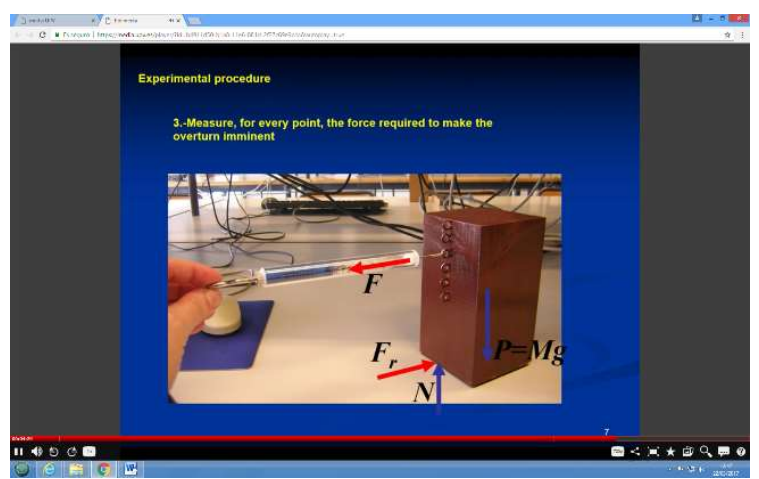

Fig. 1: video Tipo A: screencast con la voz del profesor

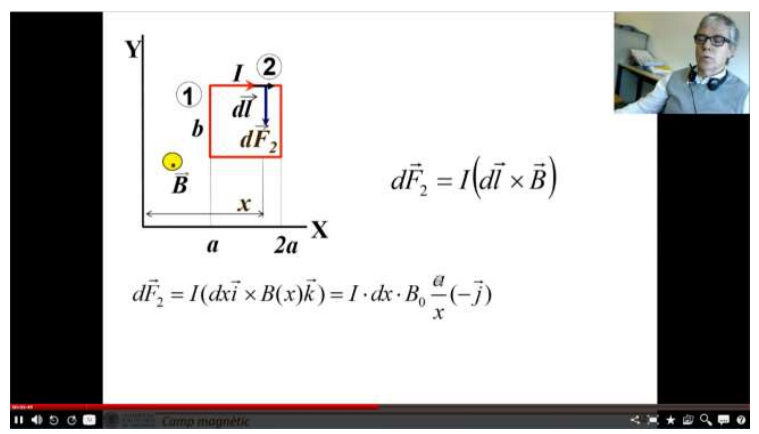

Fig. 2: video Tipo B: screencast con imagen de profesor incrustrada 


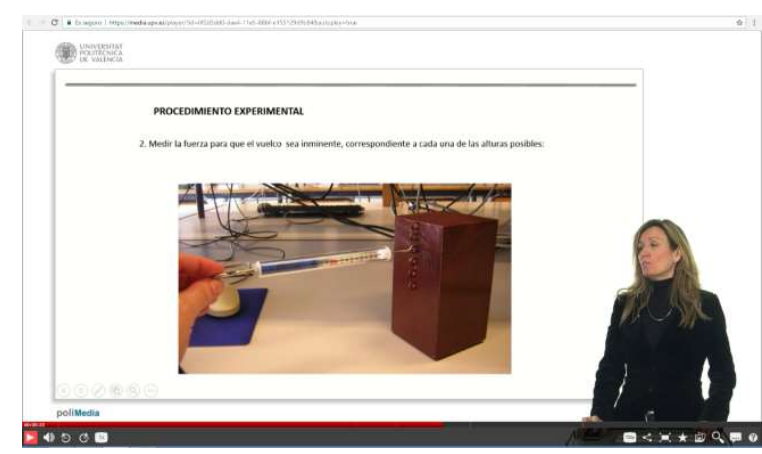

Fig. 3: video Tipo C: formato Polimedia (UPV)

La opinión del alumnado se ha obtenido mediante una encuesta realizada con la herramienta exámenes de Poli[formaT]. Las preguntas, valoradas con una escala tipo Likert de respuesta múltiple, se describen con los resultados en el siguiente apartado.

\section{Resultados y discusión}

De los 155 alumnos de la asignatura, la encuesta fue contestada por una muestra formada por 54 alumnos, obteniéndose los resultados que mostramos a continuación.

\subsection{Análisis de la opinión del alumnado a las preguntas de respuesta cerrada.}

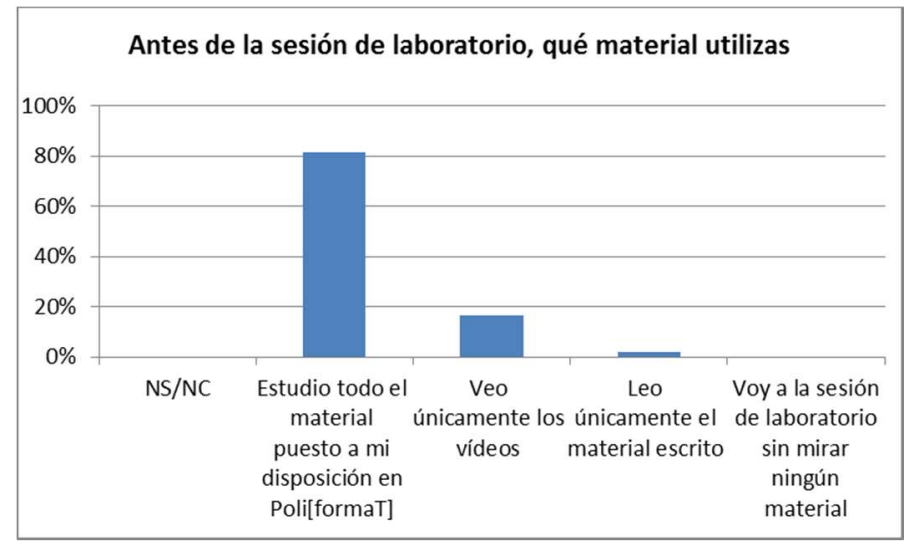

Fig.4:Material utilizado antes de la sesión de laboratorio

La Figura 4 muestra el material que el alumnado ha utilizado para la preparación de las prácticas de laboratorio, con anterioridad a la sesión. La gran mayoría estudia todo el material que ha sido puesto a su disposición, tanto audiovisual como escrito. Este resultado confirma, aunque no era objeto de estudio, que los alumnos encuestados han asimilado la metodología de "clase inversa".

La Figura 5 muestra la preferencia por parte del alumnado entre el material escrito y el material audiovisual, en el contexto de la preparación de las prácticas de laboratorio. Como se observa, prefieren el material audiovisual, según indica el porcentaje de respuestas que muestran su acuerdo (54\%). Este resultado, esperado por otro lado, no debe disuadirnos de desarrollar la competencia en comprensión lectora, tan necesaria en el contexto social actual. 


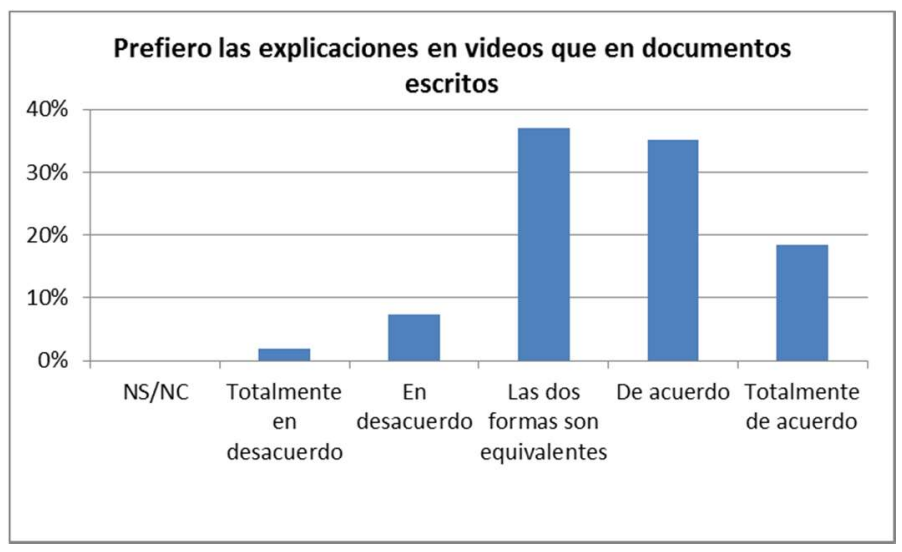

Fig.5: Preferencia entre material audiovisual o escrito

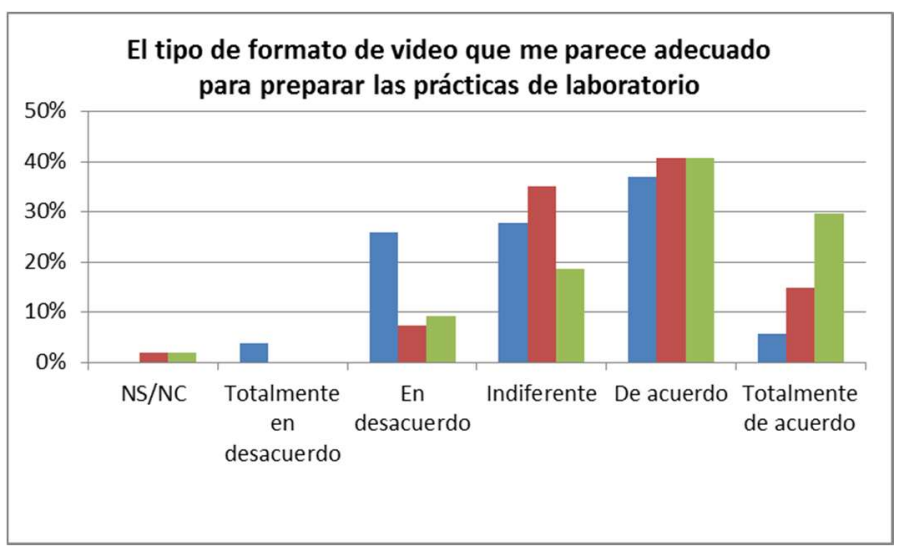

Fig. 6: Preferencia entre tipos de video tipo A (barra azul), tipo B (barra roja) y tipo C (barra verde)

En la figura 6 se puede ver la opinión por parte del alumnado sobre la adecuación de los ditintos formatos de video. Los resultados muestran que el $43 \%$ del alumnado considera adecuado el video tipo A, el 56\% el tipo B y el 70\% el Tipo C.

Se ha hecho una transformación de la escala de Likert a valores numéricos, entre 0 y 10 puntos, para obtener la media y la desviación estándar de la valoración de cada uno de los formatos de video. Los resultados se presentan en la tabla 1 .

Tabla 1. Valoración de los tres formatos de video

\begin{tabular}{ccc}
\hline & Promedio & Desviación Estándar \\
\hline Tipo A & 5,4 & 2,5 \\
Tipo B & 6,5 & 2,1 \\
Tipo C & 7,2 & 2,3 \\
\hline
\end{tabular}

Con objeto de evaluar la relevancia de estas diferencias, se ha realizado un análisis de Varianzas (ANOVA) (SPSS para Windows v.16 ) tomándose como nivel de significación, alfa=0,05. El test de Levene no muestra diferencias significativas entre

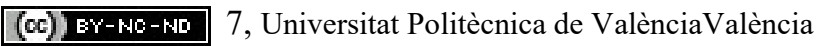


las varianzas de los diferentes grupos, por lo que se ha utilizado el método paramétrico para llevarlo a cabo. El test ANOVA mostró diferencias significativas entre las medias de los tres grupos $(\mathrm{F}=9,40 ; \mathrm{p}<0,001)$, por lo que se procedió a realizar un análisis de contrastes. El resultado obtenido fue consistente con nuestras espectativas. El análisis ANOVA obtuvo diferencias estadísticamente significativas para un contraste definido "a priori" para el vídeo tipo A con respecto al $\mathrm{B}(\mathrm{A}<\mathrm{B}$, $\mathrm{t}=2,72 ; \mathrm{p}<0,01)$ y del vídeo tipo A respecto al $\mathrm{C}(\mathrm{A}<\mathrm{C}, \mathrm{t}=4,29 ; \mathrm{p}<0,001)$, no encontrándose diferencia significativas entre el $\mathrm{B}$ y el $\mathrm{C}$.

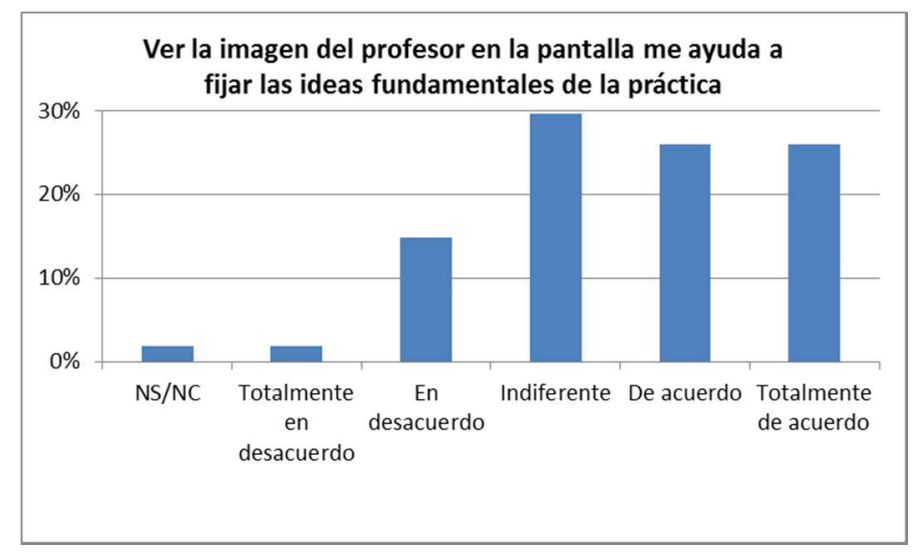

Fig. 7: Preferencia sobre la presencia de la imagen del profesor

La Figura 7 refleja la valoración de la presencia de la imagen del profesor en el material audiovisual, en el contexto de ayudar a fijar las ideas más importantes. Se observa que un $52 \%$ del alumnado la valora positivamente.

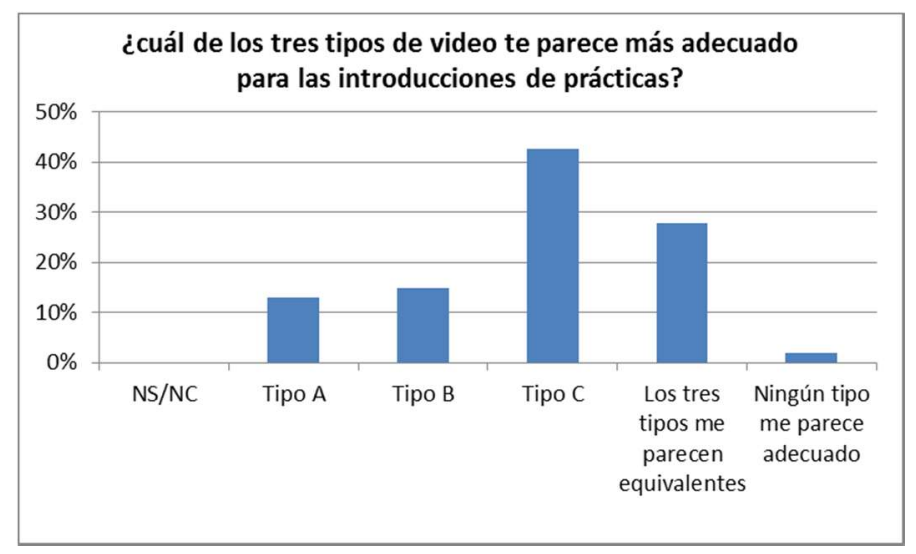

Fig. 8: Preferencia sobre el formato de video

En la Figura 8 puede verse el resultado del formato que los estudiantes consideran más apropiado, en caso de tener que elegir. Observando esta gráfica, concluimos que los alumnos prefieren el formato Tipo $\mathrm{C}$, aunque es de destacar también que hay un porcentaje muy significativo (28\%) del alumnado que considera equivalente los tres tipos. El sentido que dan a esta equivalencia se concreta con el análisis del apartado siguiente, en el que se recoge la 
respuesta abierta de los alumnos: en cierta forma la equivalencia se transforma en complementariedad.

\subsection{Análisis de la opinión del alumnado a la pregunta de respuesta abierta.}

Hemos analizado las opiniones del alumnado expresadas en la pregunta: "Añade cualquier comentario que creas conveniente sobre los distintos tipos de vídeos utilizados en la preparación de las prácticas de laboratorio. En particular nos interesa saber lo que más te gusta y lo que menos te gusta de estos tipos de vídeo". De los 54 alumnos que han contestado la encuesta, el $40 \%$ ha contestado a esta pregunta.

Destacamos las opiniones relacionadas con los contenidos del trabajo. Las opiniones de los alumnos sobre la utilización de vídeos en la presentación de prácticas, en general, es positiva: "los vídeos son una gran apuesta para la preparación de las prácticas de laboratorio", porque "se combina la explicación oral con la imagen visual, de manera que el aprendizaje es más completo". "El nivel de calidad lo considero alto". Destacan que "son concisos y claros, van directamente al grano", "el formato es el correcto, no son muy largos, en los que la gente desconecta". También destacan el problema fundamental de la docencia inversa cuando afirman que "si algún día se te olvida ver el vídeo estás totalmente perdido y no te vuelves a enganchar a la clase en toda la sesión de laboratorio".

Hay un grupo de alumnos que consideran los vídeos un complemento, o un conjunto, de la documentación escrita: "completar la información escrita con las explicaciones del profesor en algún vídeo me resulta de gran ayuda", ya que les "aportan claridad".

Cabe mencionar que, como es lógico en una metodología docente basada en la evaluación continuada y trabajo en equipo, hay alumnos con una opinión crítica y cuestionan el exceso de documentación de que disponen (vídeos, documentación escrita, para antes y durante la realización de la práctica): "llevamos exceso de información a la práctica" lo que hace que "nos cuesta más hacerlas", consultando los documentos y vídeos si "mientras la estamos realizando tenemos que volver a consultar algunos pasos". Proponen hacer "un resumen que ordenaría y aclararía las ideas".

En opinión de varios alumnos, "es preferible ver al profesor explicar", "ya que es un formato más cercano. A muchos estudiantes les reconforta la presencia de una persona que explica, la pueden ver". Ver al profesor hace "más amena la explicación". Por otro lado te "ayuda a sintetizar", "a prestar atención” y "a entender y concentrarte en las ideas fundamentales". Sin embargo, también hay una respuesta en la que consideran que "en determinadas, ocasiones la presencia del profesor puede disminuir la concentración necesaria en ejercicios complejos". Ningún alumno destaca el Tipo C de formato de vídeo, pero sí que hay opiniones que destacan el B: "En mi opinión el mejor tipo de vídeos es el B, ya que en el tipo C la imagen completa del profesor ocupa la pantalla y puede llegar a ser molesto".

En cuanto a los contenidos hay opiniones contradictorias. Mientras que alguno considera que "lo que menos me gusta es la parte excesivamente teórica que dificulta la comprensión", a otros les gustaría profundizar en temas teóricos: “dedicar más tiempo a la explicación del cálculo de incertidumbres o errores". Hay opiniones de que los vídeos deben contener ejemplos filmados de cómo realizar partes de la práctica: “a veces mostrar las herramientas

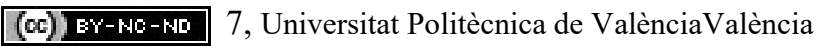


no ayuda a entender cómo usarlas", y por lo tanto "ayudaría que el profesor realizase un ejemplo práctico de las conexiones de los elementos de un circuito de medida", ya que "sólo una imagen (una fotografía) no explica bien cómo y por qué conectar" los componentes y equipos de medida.

Sobre cuestiones técnicas de los vídeos, de todos los formatos destacan los problemas relacionados con el audio: "alguns vídeos l'audio estava molt baix i no es podia entendre", "tienen defectos de sonido", "la calidad del sonido podría ser mejor". Algunos consideran que "la imagen del profesor estaría mejor fuera del espacio utilizado para explicar la práctica", y otros, aunque la herramienta de visualización de los vídeos permite acelerar hasta 1.5 la velocidad de reproducción, consideran que "algunos vídeos explican cosas de forma lenta, y es más rápido leerte la documentación escrita" y "tienes la sensación de que pierdes el tiempo".

\section{Conclusiones}

Los resultados analizados han demostrado que los estudiantes, en general, prefieren archivos de vídeo a documentos de texto para el estudio y la preparación de las prácticas de laboratorio. Esto confirma la primera hipótesis planteada.

En cuanto a los resultados acerca de los tipos de vídeos, se observa una preferencia estadísticamente significativa a favor de los videos en los que se muestra la imagen del profesor, lo cual parece confirmar la segunda hipótesis.

Aunque el formato de video Polimedia ha sido el mejor valorado, existen factores que pueden añadir ciertos matices a esta consideración. Por una parte, la valoración que hacen de los otros dos tipos de vídeos (screencast con o sin imagen del profesor) también es buena. Por otra parte, en los comentarios mostrados por los estudiantes en la pregunta de respuesta abierta resulta frecuente que el alumnado sea crítico con el formato Polimedia y que valoren positivamente el screencast con imagen del profesor. Además, resulta destacable también que hay un 30\% de alumnos que manifiestan que los tres formatos utilizados pueden ser válidos. Por lo tanto, y respecto de la tercera hipótesis planteada, quedan abiertos nuevos factores en los que profundizar, y no se puede afirmar con rotundidad que los estudiantes prefieran el formato Polimedia frente a los screencast. Posiblemente, un formato híbrido de los tres tipos conseguiría un mayor consenso.

\section{Agradecimientos}

Los autores agradecen al Instituto de Ciencias de la Educación de la universitat Politècnica de València su apoyo al grupo de Innovación e-MACAFI y al Proyecto PIME/2016/A/017.

\section{Referencias}

Ahmad, T., \& Doheny, F. (2014). Six key benefits of screencasts in learning Maths. In Recent Trends in Social and Behaviour Sciences (pp. 283-288). CRC Press. 
https://doi.org/10.1201/b16658-50

Blasco, A. C., Lorenzo, J., Sarsa, J., \& Sarsa, J. (2016). The flipped classroom and the use of educational software videos in initial teaching education. Qualitative study. @tic. RevistaD’innovacióEducativa, 0(17), 12-20. https://doi.org/10.7203/attic.17.9027

Dunn, P. K., McDonald, C., \& Loch, B. (2015). StatsCasts: screencasts for complementing lectures in statistics classes. International Journal of Mathematical Education in Science and Technology. https://doi.org/10.1080/0020739X.2014.990530

Jordan, C., Loch, B., Lowe, T., Mestel, B., \& Wilkins, C. (2012). Do short screencasts improve student learning of mathematics? MSOR Connections. https://doi.org/10.11120/msor.2012.12010011

Lasry, N., Dugdale, M., \& Charles, E. (2014). Just in Time to Flip Your Classroom. The Physics Teacher, 52(1), 34. https://doi.org/10.1119/1.4849151

Mayer, R. E. (2014). The Cambridge Handbook of Multimedia Learning (2nd Edition).

Oud, J. (2009). Guidelines for effective online instruction using multimedia screencasts. Reference Services Review, 37(2), 164-177. https://doi.org/10.1108/00907320910957206

Peterson, E. (2007). Incorporating screencasts in online teaching. International Review of Research in Open and Distance Learning, 8(3).

Sugar, W., Brown, A., \& Luterbach, K. (2010). Examining the anatomy of a screencast: Uncovering common elements and instructional strategies. International Review of Research in Open and Distance Learning, 11(3), 120.

Sutton-Brady, C., Scott, K. M., Taylor, L., Carabetta, G., \& Clark, S. (2011). The value of using short-format podcasts to enhance learning and teaching. Research in Learning Technology, 17(3), 219-232. https://doi.org/10.3402/rlt.v17i3.10878

Zaphiris, P., \& Ioannou, A. (2015). Learning and Collaboration Technologies: Second International Conference, LCT 2015 Held as Part of HCI International 2015 Los Angeles, CA, USA, August 2???7, 2015 Proceedings. Lecture Notes in Computer Science (Including Subseries Lecture Notes in Artificial Intelligence and Lecture Notes in Bioinformatics), 9192, 714-723. https://doi.org/10.1007/978-3-319-20609-7 